\title{
Caracterisation Physico-Chimique Du Lixiviat De La Decharge Sauvage De Meknes
}

\author{
Zakariae Mejraoua \\ Nasser-Eddine Zine \\ Equipe Biodiversité \& Ecologie des Zones Humides, Université Moulay \\ Ismail, Faculté des Sciences Meknès-Maroc
}

Doi: 10.19044/esj.2017.v13n33p154 URL:http://dx.doi.org/10.19044/esj.2017.v13n33p154

Abstract

Solid and urban waste produced by the municipality of Meknes amounts to more than $185.10^{3}$ tons annually. Since 2002 they have been transported to a landfill controlled, wild of an area of 23 hectares on the outskirts of the Commune. This discharge is unfortunately devoid of a leachate treatment device (brownish liquid effluent, produced under the combined action of rainwater and fermentation of buried waste). This study aims to make a physico-chemical characterization and evaluate the states mineral and organic of this leachate. It should be noted that this physicochemical characterization is arbitrary because this discharge will soon be equipped with a waste disposal and recovery center (CEV) using Evalixm as a treatment method. Since 2014, the company " SUEZ "Environment began to put in place under control this discharge. For the realization of this characterization, we carried out a series of sampling leachate from June 2014 to June 2015. During this period, the physico-chemical analyzes carried out on these samples, revealed:

- low levels of nitrates $\left(\mathrm{NO}^{3-} \max =18,1 \mathrm{mg} / \mathrm{L}\right)$ and phosphates $\left(\mathrm{PO}_{4}{ }^{3-} \max \right.$ $=0,38 \mathrm{mg} / \mathrm{L}$ )

- high levels of ammonium $\left(\mathrm{NH}_{4}{ }^{+} \max =54,13 \mathrm{mg} / \mathrm{L}\right)$ and nitrites $\left(\mathrm{NO}^{2}\right.$, $\max =27,67 \mathrm{mg} / \mathrm{L}$ ). This mineral concentration is translated by a high electrical conductivity which has reached a maximum value of $7.35103 \mu \mathrm{S}$ / $\mathrm{cm}$. Organic pollution estimated by the pollutant load is important; the values of the Calculated DCO from 1205 to $9207 \mathrm{mg} \mathrm{O}_{2} / \mathrm{L}$ with an average of $4808 \mathrm{mg} \mathrm{O} / \mathrm{L}$, those of DBO are between 53 and $386 \mathrm{mg} \mathrm{O} 2 / \mathrm{L}$ for an average of $157 \mathrm{mg} \mathrm{O} / \mathrm{L}$. The values of the ratio DBO5 / DCO oscillate between 0.01 and 0.08 with an average of 0.04 . This which testifies to the anaerobic character that prevails at the level of the discharge and a probable contamination of the water table. The hydrogen variant $(\mathrm{pH})$ between 6,42 and 8,99 is an annual average of 8,42 which shows that the leachate 
produced by the landfill is basic. The $\mathrm{pH}$ value shows a stagnation (8.42 and 8.23) from one year to the next, reflecting its stability and aging

Keywords: Leachate -discharge - physico-chemistry - Meknes.

\section{Resume}

Les déchets solides et urbains produits par la Commune de Meknès s'élèvent à plus de $185.10^{3}$ tonnes annuellement. Ils sont véhiculés depuis 2002 vers une décharge non contrôlée, sauvage d'une superficie de $\mathbf{2 3}$ hectares à la périphérie de la Commune. Cette décharge est malheureusement dépourvue d'un dispositif de traitement du lixiviat (effluent liquide brunâtre produit sous l'action conjuguée de l'eau de pluie et de la fermentation des déchets enfouis). Cette étude a pour but de faire une caractérisation physico-chimique et d'évaluer les états minéral et organique de ce lixiviat.Il est à signaler que cette caractérisation physico-chimique est arbitraire car cette décharge sera bientôt équipée d'un centre d'élimination et de valorisation des déchets (CEV) utilisant Evalixm comme procédé de traitement. En effet depuis 2014, la société 'SUEZ', Environnement a commencé à mettre en place sous contrôle cette décharge. Pour la réalisation de cette caractérisation, nous avons procédé à une série d'échantillonnage du lixiviat de juin 2014 à juin 2015. Au cours de cette période, les analyses physico-chimiques effectuées sur ces échantillons, ont révélé :

teneurs faibles en nitrates $\left(\mathbf{N O}_{3}-\mathbf{m a x}=\mathbf{1 8}, \mathbf{1} \mathbf{~ m g} / \mathrm{L}\right)$ et en phosphates $\left(\mathbf{P O}_{4}{ }^{{ }^{-}}\right.$ $\max =0,38 \mathrm{mg} / \mathrm{L}$ )

- des teneurs élevés d'ammonium $\left(\mathbf{N H}_{4}{ }^{+} \mathbf{m a x}=\mathbf{5 4 , 1 3} \mathbf{~ m g} / \mathbf{L}\right)$ et en nitrites $\left(\mathrm{NO}_{2}-, \mathbf{m a x}=\mathbf{2 7 , 6 7} \mathrm{mg} / \mathrm{L}\right.$. Cette concentration minérale est traduite par une forte conductivité électrique qui atteint une valeur maximale de $\mathbf{7 , 3 5}$ $\mathbf{1 0}^{3} \boldsymbol{\mu S} / \mathbf{c m}$. Les valeurs de la DCO enregistrées varient de 1205 à $9207 \mathbf{~ m g}$ D'O $/ 2$ avec une moyenne de $4808 \mathrm{mg} \mathrm{D'} \mathbf{O}_{2} / \mathrm{L}$, celles de la $\mathrm{DBO}_{5}$ sont comprises entre 53 et $386 \mathrm{mg} \mathrm{D}^{\prime} \mathrm{O}_{2} / \mathrm{L}$ pour une moyenne de $157 \mathrm{mg}$ D'O $2 /$ L. Les valeurs du rapport DBO5/DCO oscillent entre $\mathbf{0 , 0 1}$ et $\mathbf{0 , 0 8}$ avec une moyenne de $\mathbf{0 , 0 4}$. Ce qui témoigne le caractère anaérobique qui règne au niveau de la décharge et d'une probable contamination de la nappe phréatique. Le potentiel d'hydrogène $(\mathbf{p H})$ variant entre 6,42 et 8,99 soit une moyenne annuelle de $\mathbf{8 , 4 2}$ ce qui montre que le lixiviat produit par la décharge est basique. La valeur du pH montre une stagnation $(\mathbf{8 , 4 2}$ et $\mathbf{8 , 2 3}$ ) d'une année à une, ce qui témoigne de sa stabilité et de son vieillissement.

Mots clés : Lixiviat- Décharge-physico-chimique-Meknès

\section{Contexte de l'étude}

$\mathrm{Au}$ Maroc, la production des déchets solides est en augmentation 
constante, suite à la croissance démographique et à l'intensification de l'activité économique. La mise en décharge non contrôlée à ciel ouvert des déchets reste le moyen le plus utilisée surtout dans les pays en voie de développement, d'après Saadi et al 2013.

Plusieurs programmes, au Maroc sont en cours de réalisation en matière d'assainissement solide et liquide. Parmi ces programmes, celui sur les déchets ménagers (PNDM) qui consiste à la mise en place des décharges contrôlées et à la réhabilitation des décharges sauvages (Ministère délégué auprès du Ministre de l'Energie, des Mines, de l'Eau et de l'Environnement, chargé de l'Environnement,2004).

L'un des impacts de la mise en décharge est la production d'effluents liquide riches en matière organiques et minérales, et métalliques appelés lixiviats. Ces derniers proviennent de la percolation des eaux de pluies à travers les dépôts, et constituent une source de pollution (Hakkou et al 2001).

Au Maroc les études menées par Saadi et al en 2013 sur les lixiviats du centre d'Enfouissement (CET) de la ville d'Oujda ont révélé de fortes teneurs en matières organiques biodégradables, en métaux lourds, en matière minérales et en suspension.

En Algérie, les travaux de Mokhtaria et al. En 2007 sur la décharge de Tiaret a montré que les lixiviats renferment de nombreux contaminants organiques et métalliques $(\mathrm{Pb}, \mathrm{Zn}, \mathrm{Cd}, \mathrm{Ni}, \mathrm{Cr})$ supérieures aux normes admises. Ceux de Bennama et al. En 2010 sur la décharge d'El Kerma ont montré des résultats similaires.

En côte d'Ivoire, Kouamé (2007), à partir de modèle mathématique, a suivi l'évolution des lixiviats de la décharge d'Akouédo et évaluer leur impact sur la qualité des eaux souterraines.

Toujours en côte d'Ivoire, les travaux d'Adjiri en 2008 ont permis de caractériser la pollution chimique et microbiologique de l'environnement d'Akouédo afin de quantifier les risques sanitaires afférents à cette pollution.

Problématique : Le traitement des ordures ménagères, reste très peu développé en dehors de la mise en décharge non contrôlée, quasi-généralisée par les communes marocaines.

Cette pollution engendrée par les lixiviats non traités est la conséquence d'importants dommages sur l'environnement (pollution des eaux souterraines et superficielles, pollution des sols, impact sur la santé humaine, émission des Gaz à effet de serre...)

Objectif de l'étude : Cette étude a donc pour but de lutter contre la pollution causée cet lixiviat non traité, après avoir réalisé une caractérisation physico-chimique.

\section{Localisation de la décharge}

La décharge publique de Meknès (Fig 1) se situe dans le bassin 
versant du R'Dom comprenant les oueds : Boufekrane, Ouislane et Serja, le plus important de ces Oueds est celui de Boufekrane où le lixiviat se déverse directement.

La topographie du site actuel de la décharge (23 ha) est ondulée, le site de la décharge est une vallée de 409 mètre d'altitude, la morphologie des terrains aux alentours, montrent des pentes fortes au Nord Ouest alors que les faibles pentes sont du coté Nord Est. Le réseau hydrographique présente une direction qui coïncide avec la direction de la surface structurale SSE-NNW d'après (Essahlaoui A., 2000).

\section{Echantionnage et analyse}

De juin 2014 à juin 2015 une compagne d'échantillonnage par mois a été réalisée à la décharge de Meknès, afin de faire un suivi annuel et interannuel, des paramètres physiques, minérales et organiques.

Les prélèvements ont été effectués à l'aide d'un flacon de $500 \mathrm{ml}$ ou une perche télescopique, fixé d'un flacon de $500 \mathrm{ml}$ à son extrémité (Rodier et al, 2009).

Les échantillons de lixiviats ont été prélevés dans des bouteilles en polyéthylène de capacité $1 \mathrm{~L}$, préalablement lavées à l'acide nitrique puis à l'eau distillée (Rodier et al, 2009).

Les échantillons de lixiviat ont été conservés dans une glacière pendant le transport au laboratoire $\left(+4^{\circ} \mathrm{C}\right)$.

Le dosage de ces paramètres a été réalisé en se référant aux méthodes d'analyses chimiques décrites par Rodier et al (2009). 


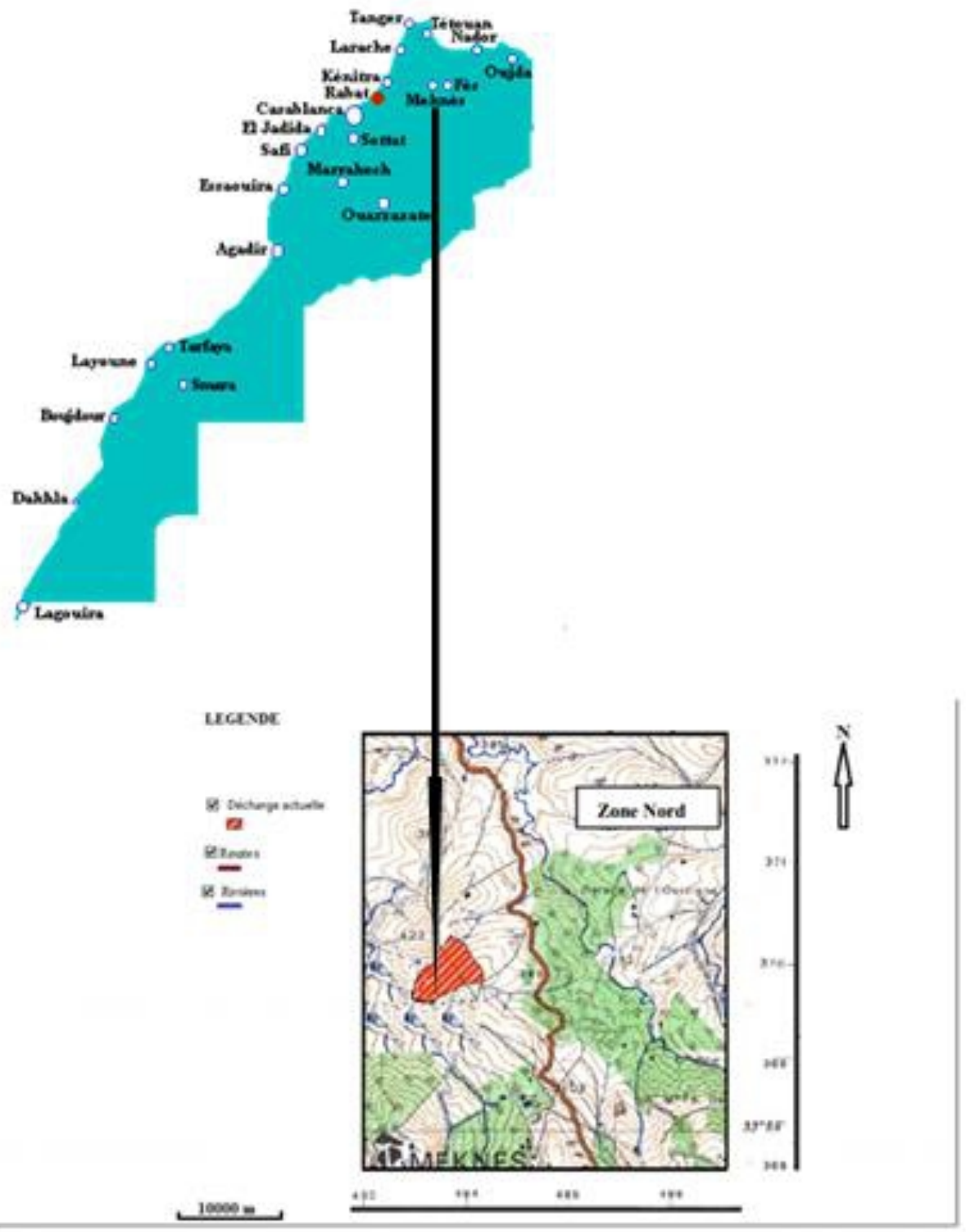

Figure 1 : Carte du Maroc et localisation du site de la décharge (Chalabi M., Benmbarek M., Margaa K.2001). 


\section{Resultats et discussion}

\section{Paramètres physiques}

tab.1.

Les résultats des analyses physico-chimiques sont présentés dans le

Tableau 1 : Caractéristiques physico-chimique des lixiviats de la décharge de Meknès

\begin{tabular}{cccccc}
\hline Variables & Minimum & Maximum & Moyenne & $\begin{array}{c}\text { Ecart- } \\
\text { type }\end{array}$ & Unité \\
\hline Conductivité & $2,73.10^{3}$ & $7,35.10^{3}$ & $5,12.10^{3}$ & $1,6.10^{3}$ & $\underline{\underline{\mu} \mathrm{S} / \underline{\mathrm{Cmcm}} \mathrm{à} 25}$ \\
Température & 15 & 30 & 21.85 & 5 & ${ }^{\circ} \mathrm{C}$ \\
$\mathrm{DBO}$ & 53 & 386 & 157.18 & 90.60 & ${ }^{\circ} \mathrm{C}$ \\
$\mathrm{DCO}$ & 1205 & 9207 & 4808,10 & 1939,13 & $\mathrm{mgO}_{2} / \mathrm{L}$ \\
$\mathrm{MES}$ & 893 & 4610 & 2543,05 & 1373,86 & $\mathrm{mg} / \mathrm{L}$ \\
$\mathrm{NO}^{2-}$ & 0,09 & 27,67 & 16,47 & 7,64 & $\mathrm{mg} / \mathrm{L}$ \\
$\mathrm{NO}^{3-}$ & 9,04 & 18,1 & 14,17 & 3,14 & $\mathrm{mg} / \mathrm{L}$ \\
$\mathrm{SO}_{4}{ }^{2-}$ & 5,75 & 600,5 & 142,85 & 178,93 & $\mathrm{mg} / \mathrm{L}$ \\
$\mathrm{pH}^{-}$ & 6,42 & 8,99 & 8,24 & 0,74 & - \\
$\mathrm{NH}_{4}^{+}$ & 34.11 & 54,13 & 44,86 & 6,29 & $\mathrm{mg} / \mathrm{L}$ \\
$\mathrm{PO}_{4}^{-}$ & 0 & 0,38 & 0,10 & 0,15 & $\mathrm{mg} / \mathrm{L}$ \\
\hline
\end{tabular}

Sur la base des résultats du tableau 1 nous constatons que le $\mathrm{pH}$ du lixiviat varie de 6,42 à 8,99 avec une moyenne de 8,24, sachant que les valeurs limite de référence sont comprises entre 3.7 et 8.8. Le potentiel d'hydrogène $(\mathrm{pH})$ est légèrement neutre à alcalin pour la décharge de Meknès, ce caractère basique a été trouvé par Kouadio et al. (2000), Kouamé (2007), pour la décharge d'Akouedo, en Côte d'Ivoire (tab 2).

Ces valeurs sont de mêmes ordres de grandeur que celles obtenues par El Khamlichi et al. (1997) pour la décharge de Rabat (Maroc).

Les valeurs de $\mathrm{pH}$ obtenues dans les lixiviats pourraient être liées à la faible concentration de composés organiques volatiles. En effet, lors de la fermentation acide, première phase de la décomposition anaérobie des déchets, les percolats jeunes sont riches en composés organiques volatiles. $\mathrm{Au}$ cours de cette phase, les valeurs de $\mathrm{pH}$ enregistrées sont généralement inférieures à 4 d'après Tchobanoglous et $a l$., en 1993.

$\mathrm{Au}$ fur et à mesure du vieillissement de la décharge, le lixiviat s'appauvrit en composés organiques volatiles. Ceci va alors entraîner une montée du pH à 7 ou plus (Kjeldsen et al., 2002). 
Tableau 2 : Caractéristiques physico-chimiques de lixiviats de la décharge de Meknès en comparaison avec quelques données bibliographiques

\begin{tabular}{|c|c|c|c|c|c|c|c|}
\hline \multirow[b]{2}{*}{ Paramètres } & \multirow[b]{2}{*}{$\begin{array}{c}\text { Notre } \\
\text { zone } \\
\text { d'étude } \\
\text { Meknes } \\
2015\end{array}$} & \multicolumn{6}{|c|}{ Comparaison avec des études antérieures } \\
\hline & & $\begin{array}{c}\text { Akouedo } \\
2014\end{array}$ & $\begin{array}{c}\text { Akouedo } \\
\text { (Kouadio } \\
e t \\
\text { al., 2000) }\end{array}$ & $\begin{array}{c}\text { Akouedo } \\
\text { (Kouamé, } \\
\text { 2007) }\end{array}$ & $\begin{array}{c}\text { Rabat } \\
\text { (El } \\
\text { Khalmic } \\
\text { hi et al., } \\
\text { 1997) }\end{array}$ & $\begin{array}{c}\text { Tiaret } \\
\text { (Mokhtaria } \\
\text { et al.,2007) }\end{array}$ & $\begin{array}{l}\text { Oujda } \\
\text { (Saadi } \\
\text { et al., } \\
\text { 2013) }\end{array}$ \\
\hline $\mathbf{P H}$ & $\begin{array}{l}6,42- \\
8,99\end{array}$ & $7,04-8,76$ & $9,6-9,8$ & $7,81-8,11$ & $7-8$ & - & $\begin{array}{c}5,59- \\
6,08\end{array}$ \\
\hline $\mathbf{T}\left({ }^{\circ} \mathrm{C}\right)$ & $15-30^{\circ} \mathrm{c}$ & $28,4-32,7$ & $24,1-28,4$ & $\begin{array}{c}33,70- \\
39,5\end{array}$ & - & - & $\begin{array}{c}14,90- \\
15,70\end{array}$ \\
\hline $\mathrm{CE}(\mu \mathrm{S} / \mathrm{cm})$ & $\begin{array}{c}2,73 \cdot 10^{3}- \\
7,35 \cdot 10^{3}\end{array}$ & $\begin{array}{l}2390- \\
17150\end{array}$ & - & $375-7770$ & $\begin{array}{c}23130- \\
74600\end{array}$ & - & $\begin{array}{l}14,84- \\
37,60\end{array}$ \\
\hline $\begin{array}{c}\mathrm{DCO} \\
\left(\mathrm{mg} / \mathrm{O}_{2} / \mathrm{L}\right)\end{array}$ & $\begin{array}{l}1205- \\
9207\end{array}$ & $642-2739$ & $310-2495$ & $956-2189$ & $\begin{array}{c}260- \\
50112\end{array}$ & $780-1230$ & $\begin{array}{l}68036- \\
89472\end{array}$ \\
\hline $\begin{array}{c}\mathrm{DBO}_{5} \\
\left(\mathrm{mg} / \mathrm{O}_{2} / \mathrm{L}\right)\end{array}$ & $53-386$ & $300-1054$ & $186-1488$ & $382-1150$ & $5-10500$ & $135-200$ & $\begin{array}{c}38188- \\
70682\end{array}$ \\
\hline
\end{tabular}

Source : A.E. Kouassi et al. / Larhyss Journal, 19 (2014)

Par ailleurs, les températures enregistrées oscillent entre $15{ }^{\circ} \mathrm{C}$ et $30^{\circ} \mathrm{C}$. Ces valeurs seraient favorables au maintien de colonies de microorganismes "mésophiles" qui se développent à une température comprise entre $20^{\circ} \mathrm{C}$ et $40^{\circ} \mathrm{C}$ (Kouamé, 2007).

Quant à la conductivité électrique, les valeurs s'échelonnent entre $2,7310^{3} \mu \mathrm{S} / \mathrm{cm}$ et $7,3510^{3} \mu \mathrm{S} / \mathrm{cm}$. La valeur moyenne est de $5,1210^{3}$ $\mu \mathrm{S} / \mathrm{cm}$. Les valeurs limites de référence se situent entre $1410^{5}$ et $17.110^{3}$ $\mu \mathrm{S} / \mathrm{cm}$

Les fortes valeurs de la conductivité ont été enregistrées en été et au printemps, périodes secs $\left(6.8810^{3} \mu \mathrm{S} / \mathrm{cm}\right.$ et $\left.5.9110^{3} \mu \mathrm{S} / \mathrm{cm}\right)$, par contre les faibles valeurs en automne et en hiver (périodes humides et pluvieuses) (3.72 $\mu \mathrm{S} / \mathrm{cm}$ et $4.64 \mu \mathrm{S} / \mathrm{cm}$ ). Revoir vos phrases. Ces faibles valeurs pourraient s'expliquer par le phénomène de dilution. En effet, pendant la saison des pluies, le lixiviat reçoit une quantité importante d'eau entraînant une dilution considérable des éléments chimiques en présence (Kouamé, 2007).

\section{Analyse des sels nutritifs}

Azote ammoniacal (NH4+), C'est un gaz soluble dans l'eau. Les teneurs varient entre 34,11 et $48,01 \mathrm{mg} / \mathrm{L}$ en période de pluie et entre 54,13 et $41,09 \mathrm{mg} / \mathrm{L}$ en période sèche. Les valeurs limites de référence se situent entre 2 et $3870 \mathrm{mg} / 1$. Cet élément chimique est le principal agent réducteur 
dans le lixiviat, et il constitue à long terme un polluant important (Christensen et al., 2001)

Nitrates (NO3-) et nitrites (NO2-) : les nitrates constituent le stade final de l'oxydation de l'azote, et représentent la forme d'azote au degré d'oxydation le plus élevé présent dans l'eau. Les teneurs enregistrées oscillent entre à 9,04 et $18,1 \mathrm{mg} / \mathrm{L}$ avec une teneur moyenne de $14.17 \mathrm{mg} / \mathrm{L}$. Cette valeur est largement inférieure à la concentration maximale admise par l'OMS (1980) qui est de $50 \mathrm{mg} / \mathrm{L}$. Les valeurs limites de référence se situent entre 2 et $845 \mathrm{mg} / \mathrm{l}$.

Les nitrites proviennent, soit d'une réduction des nitrates, soit d'une oxydation incomplète des ions ammonium (Rodier et al ,2009). Les teneurs en $\mathrm{NO}_{2}{ }^{-}$des lixiviats sont faibles et sont comprises entre 0,09 et $27,67 \mathrm{mg} / \mathrm{L}$, avec une moyenne de $16,47 \mathrm{mg} / \mathrm{L}$. Ces faibles teneurs sont dues aux valeurs faibles de l'oxygène dissous et du potentiel redox dans le lixiviat.

Ortho phosphates $\left(\mathrm{PO}_{4}{ }^{3-}\right)$ pour ce paramètre, l'analyse des résultats montre que les concentrations en $\mathbf{P O}_{4}{ }^{3-}$ dans le lixiviat oscillent entre 0 et $0,38 \mathrm{mg} /$. Les valeurs limites de référence se situent entre 0,16 et $145 \mathrm{mg} / \mathrm{l}$.

Sulfates SO4 L'augmentation des concentrations en SO4 dans notre lixiviat est due essentiellement à l'apport de cet élément contenu dans les minéraux des différentes roches sédimentaires (Gypses) par la dissolution du sulfate de magnésium dans les roches et certaine dans les terrains gypsifères et les rejets des lixiviats de la décharge. Les valeurs élevées en sulfates peuvent avoir comme origine les fertilisants agricoles.

Matières en suspension (MES) notre lixiviat présentent des valeurs de matières en suspension (MES) très importantes. Il s'agit d'un caractère commun à toutes les décharges d'ordures ménagères (Christensen P., 2001). Les fortes teneurs en (MES) s'expliquent d'une part que pendant la période estivale (maximas des températures), il y a une absence quasi-totale des précipitations qui peuvent induire des phénomènes de dilution du jus de décharge, d'où les lixiviats deviennent de plus en plus riches en MES.

\section{Paramètres organiques/}

\section{Demande Chimique en Oxygène (DCO)}

La DCO représente la quantité d'oxygène consommée par les matières oxydables chimiquement contenues dans l'eau. Elle est représentative de la majeure partie des composés organiques et des sels minéraux oxydables (BECHAC.J. P et al,1984). Les teneurs enregistrées sont comprises entre 1205 et $9207 \mathrm{mg} \mathrm{D} \mathrm{O}_{2} / \mathrm{L}$. La teneur moyenne est de $4808 \mathrm{mg} \mathrm{D} \mathrm{O}_{2} / \mathrm{L}$. Les valeurs extrêmes de référence se situent entre 31 et $10^{4} \mathrm{mg} \mathrm{D} \mathrm{O}_{2} / \mathrm{L}$. 
La valeur élevée de la DCO indique une charge organique très élevée et montrent que ces effluents liquides se trouvent dans les conditions réductrices (baisse de l'oxygène).

L'absence d'Oxygène dissous dans le lixiviat et les faibles valeurs du potentiel d'oxydo-réduction indique que le phénomène d'anaérobiose est prédominant dans la décharge de Meknès. En effet, l'oxygène est fortement sollicité pour la dégradation de la matière organique et l'oxydation des minéraux présents dans l'effluent.

Les valeurs obtenues au niveau de la charge polluante exprimée par la DCO, sont comparables à celles rapportées par El Khalmichi et al., (1997) à Rabat. Elles sont en revanche très supérieures à celles obtenues par Saadi et al. (2013) à Oujda au Maroc.

Cette différence pourrait être liée à l'âge, la nature et la quantité des déchets ainsi que les différents facteurs climatiques tels que la pluviométrie, l'humidité de l'air et la température. En effet, selon Christensen et al. (2001), ces différents facteurs sont à la base de la variabilité des charges polluantes.

\section{Demande Biochimique en Oxygène (DBO5)}

La DBO5 est un indicateur de la pollution organique des eaux. Elle exprime le niveau de biodégradabilité de l'effluent (BECHAC.J. P et al,1984). La DBO5 varient de 53 à $386 \mathrm{mg}$ d'O2/L. La valeur moyenne est d'environ $157 \mathrm{mg}$ D'O2/L. Les valeurs limites de référence se situent entre 2 et $90.103 \mathrm{mg} \mathrm{D}$ 'O2/L.

Les fractions organiques (DBO5/DCO) renseignent sur la biodégradabilité des molécules organiques et l'âge relatif du lixiviat étudié.

Les valeurs des rapports DBO5 / DCO sont comprises entre 0,01 et 0,08 avec une moyenne de 0,04 . Ces valeurs nous montrent que le lixiviat de la décharge de Meknès est riche $\mathrm{n}$ en matières organiques biodégradables.

Ainsi, Selon Amokrane (1994, Tab 3) :

- DBO5 / DCO est supérieur à 0,5 : le lixiviat est qualifié de jeune ;

- DBO5 / DCO est compris entre 0,1 et 0,5 : le lixiviat est intermédiaire ;

- DBO5 / DCO est inférieur à 0,1 : le lixiviat est stabilisé ;

La valeur de 0,02 issue de la moyenne des différents rapports DBO5 / DCO indique que pour le lixiviat étudié est qualifié de stabilisé dans une décharge de plus de 10 ans, et caractérisé par une forte charge organique, composée essentiellement de substances humiques (acides fulviques et humiques) qui sont réfractaires à la biodégradation. Cela signifie donc, que l'état de ce lixiviat correspond à la phase de maturation de la décharge. Les valeurs limites de référence se situent entre 2 et $90.103 \mathrm{mg}$ D’O2/L 
Tableau 3 : Caractéristiques principales des différents types de lixiviats (Rapport rédigé à la demande des ministères respectivement chargés de l'environnement et de la santé Françaises (2004) :

\begin{tabular}{|c|c|c|c|}
\cline { 2 - 4 } \multicolumn{1}{c|}{} & Lixiviats jeunes & $\begin{array}{c}\text { Lixiviats } \\
\text { intermédiaires }\end{array}$ & Lixiviats stabilisés \\
\hline Age de la décharge & $<5$ ans & 5 à 10 ans & $>10$ ans \\
\hline $\mathrm{pH}$ & $<6,5$ & 6,5 à 7,5 & $>7,5$ \\
\hline $\mathrm{DCO}\left(\mathrm{g} \mathrm{L}^{-1}\right)$ & 10 à 20 & 5 à 10 & $<5$ \\
\hline $\mathrm{DBO} 5 / \mathrm{DCO}$ & $>0,5$ & 0,1 à 0,5 & Très faible \\
$($ biodégradabilité $)$ & bonne & Assez faible & $<5$ \\
\hline $\mathrm{AGV}(\%$ du COT $)$ & $>70$ & 5 à 30 & $<$ \\
\hline
\end{tabular}

$\mathrm{AGV}^{*}$ : acides gras volatils.

\section{Conclusion}

Au terme de cette étude, il ressort que :

-après plus de 10 ans d'exploitation la décharge sauvage de Meknès, rentre dans une phase de méthanogène $(\mathrm{DBO} / \mathrm{DCO}=0.04)$ qui est réalisée par des microorganismes anaérobiques stricts. Elle est ainsi considérée comme décharge stabilisée, état qui est confirmé par son $\mathrm{pH}$ moyen annuel basique : 8.24

-Son lixiviat est ainsi caractérisé par une forte charge organique avec une DCO qui dépasse la valeur de référence, charge non ou faiblement dégradable composée essentiellement de substances humiques (acides fulviques et humiques).

-La pollution minérale, est traduite par les valeurs élevées dépassant les valeurs limites de référence : $\mathrm{NH} 4+(54,13 \mathrm{mg} / \mathrm{L})$, et NO2- $(27.67 \mathrm{mg} / \mathrm{L})$, et NO3- $(18.1 \mathrm{mg} / \mathrm{L})$ et les ions $\mathrm{PO} 43-(0,38 \mathrm{mg} / \mathrm{L})$, ce qui indique bien l'importance des charges minérales et organiques décrites précédemment.

-Cette pollution minérale est traduite par une forte conductivité électrique qui atteint une valeur de $7.35103 \mu \mathrm{S} / \mathrm{cm}$.

-L'état actuel de cette décharge non contrôlée présente des risques potentiels de dégradation de l'environnement (production des biogaz);

-Cette décharge doit être réhabilitée dans l'immédiat, d'après Tahiri et al, (2014) la contamination de la nappe est retardée par la présence des marnes sur lesquelles repose la décharge à ciel ouvert non contrôlée ;

-En attendant, le fonctionnement de la décharge contrôlée, il est important de mettre en place un système de récupération des lixiviats produits par les déchets. 


\section{References:}

1. AHEL M., MIKAC N., COSOVIC B., PROHIC E., SOUKUP V. (1998). The impact of contaminant from a municipal solid waste landfill (Zagreb, Croatie) on underlying soil, Water Sci. Technol., Vol 37, ${ }^{\circ} 8,203-210$.

2. AMOKRANE A. (1994). Epuration des lixiviats de décharges. Prétraitement par coagulation-floculation. Traitement par osmose inverse. Post-traitement par incinération. Thèse de Doctorat, INSA de Lyon, France, 286 pages

3. ADJIRI O.A., GONE D.L., KOUAME I.K., KAMAGATE B., BIEMI J. (2008). Caractérisation de la pollution chimique et bactériologique de l'environnement de la décharge d'Akouedo Abidjan-Côte d'Ivoire, International journal of Biological and Chemical Scientific, Vol. 2, n4, 401-410.

4. BECHAC.J. P, BOUTIN.P, NUER. P, Traitement des eaux usées. Ed. Eyrolls. Paris 1984.

5. BENNAMA T., YOUNSI A., ZOUBIR D., DEBAB A. (2010). Caractérisation et traitement physico-chimique des lixiviats de la décharge publique d'El- Kerma (Algérie) par adsorption en discontinu sur de la sciure de bois naturelle et activée chimiquement, Water Qual. Res. J. Can. Vol 45, n¹, 81-90pp.

6. CHRISTENSEN P., 2001. Biogeochemistry of landfill leachate plumes. Application Geochemistry 16, 659-718.

7. CHRISTENSEN T.H., KJELDSEN P., BJERG P.L., JENSEN D.L. CHRISTENSEN J.B., BAUM A., ALBRECHTSEN H.J., HERON G. (2001). Biogéochemistry of landfill leachate plumes, Appl. Geochem., Vol 16, 659-718.

8. CHALABI M, BENMBAREK M, MAGAA K (2001) -. Rapport sur l'élimination des déchets solides de la ville de Meknès, aspects environnementaux. Royaume du Maroc, ministère de l'aménagement du territoire, de l'urbanisme, de l'habitat et de l'environnement. Département de l'environnement secrétariat général.

9. CHOFQI Amina, YOUNSI Abdelkader, LHADI El Kbir, MANIA Jacky, MURDY Jacques et VERON Alain (2007) - Lixiviat de la décharge publique d'El Jadida (Maroc): Caractérisation et étude d'impact sur la nappe phréatique. Revue Francophone d4colofie Industrielle-Trimestriel $\mathrm{N}^{\circ} 46$.

10. EL KHAMLICHI M.A., LAKRABNI S., KABBAJ M., JARBY E., KOUHEN M. (1997). Etude d'impact de la décharge d'Akrach (Rabat, Maroc) sur la qualité des ressources en eau, Revue Marocaine Civil, $\mathrm{n}^{\circ} 68,17-31$. 
11. ESSAHLAOUI A., (2000) Contribution à la reconnaissance des formations aquifères dans le bassin de Meknès- Fès (Maroc). Thèse de Doctorat, Ecole Mohammedia des ingénieurs, Rabat, Maroc, (2000) .

12. HAKKOU R. (2001). La décharge publique de Marrakech : caractérisation des lixiviats, étude de leur impact sur les ressources en eau et essai de leur traitement. Thèse de doctorat d'etat, Université de CADDI AYYAD, Maroc, $150 \mathrm{p}$.

13. HAKKOU R., WAHBI M., BACHNOU A., ELAMARI K., HANICH., HIBTI M. (2001). Impact de la décharge publique de Marrakech (Maroc) sur les ressources en eau, Bull. Eng. Geol. Env., 60, 325-336.

14. KJELDSEN P., BARLAZ M.A., ROOKER A.P., BAUM A., LEDIN A., CHRISTENSEN T.A. (2002). present and long-term composition of MSW landfill leachate, Crit. Rev., Environ. Sci. Technol., Vol. 32, $\mathrm{n}^{\circ} 4,297-336$.

15. KOUAME K.I. (2007).: Pollution physico-chimique des eaux dans la zone de la décharge d'Akouedo et analyse du risque de contamination de la nappe d'Abidjan par un modèle de simulation des écoulements et du transport des polluants. Thèse de Doctorat, Université d'Abobo Adjamé, Côte d'Ivoire, $212 \mathrm{p}$.

16. KOUADIO G., DONGUI B., TROKOUREY A. (2000). Détermination de la pollution chimique des eaux de la zone de la décharge d'Akouedo (Abidjan-Cote d'Ivoire). Revue des Sciences et Technologie, ENS-CI. Série A-01, 34-41.

17. LEKHEL Hicham, BOUCHELTA Aziz, BENZOUGAGH Brahim, MIMICH Khalid (2016) Lixiviats Issus De La Décharge Publique De La Ville De Meknès : Calcul Et Résultat Du Bilan Hydrique. Européen Scientific Journal Octobre édition vol.12, No.29 ISSN : 1857 - 7881 (Print) e - ISSN 1857- 7431.

18. MAKHOUKH M., SBAA M., BERRAHOU A., VAN CLOUSTER M. (2011). Contribution à l'étude physico-chimique des eaux superficielles de l'Oued Moulouya (Maroc oriental), Larhyss journal, ISSN 1112-3680, $\mathrm{n}^{\circ} 09,149-169$.

19. MOKHTARIA M.M., EDDINE B.B., LARBI D., AZZEDINE H., RABAH L. (2007). Caractéristiques de la décharge publique de la ville de Tiaret et son impact sur la qualité des eaux souterraines, Courrier du savoir, $n^{\circ} 8$ : 93-99.OMS (1980). Nitrates, Nitrites et composés N-nitroso, Génève, $112 \mathrm{p}$.

20. RODIER J. (1996). L'analyse de l'eau naturelle, eaux résiduaires, eau de mer, 8ème Edition, Dunod, Paris, 1383 p.

21. 13-SAADI S., SBAA M., EL KHARMOUZ M. (2013). 
Caractérisation physico- chimique de lixiviats du centre d'enfouissement technique de la ville d'Oujda (Maroc oriental), Science Lib., Editions Mersenne, Vol. 5, ${ }^{\circ}$ 130517, ISSN 21114706, 1-12.

22. SANE Y. (2002). La gestion des déchets à Abidjan : Un problème récurrent et apparemment sans solution, AJEAM/RAGEE, Vol 4, $\mathrm{n}^{\circ} 1,13-22$.

23. TAHIRI A.A; LAZIRI F; YACHAOUI Y ; JAFARI S ; TAHIRI A.H (2014) -Etude des polluants contenus dans les lixiviats issus de la décharge publique de la ville de Meknès. Européen Scientifique Journal. Déc. 2014, édition Vol 10, n³5, pp. 170-186.

24. TCHOBANOGLOUS G., THEISEN H., VIGIL S. (1993). Integrated Solid Waste Management, McGraw-Hill International Edition, New York, $978 \mathrm{p}$. 